\title{
Liposomal bupivacaine for prolonging analgesia with TAP blocks-two steps forward, one step back
}

\author{
Yatish S. Ranganath, MBBS MD - Rakesh V. Sondekoppam, MBBS MD
}

Received: 29 October 2020/ Accepted: 1 November 2020/Published online: 21 January 2021

(C) Canadian Anesthesiologists' Society 2021

Strategies to selectively prolong the duration of sensory blockade have been a subject of both controversy and intense focus in regional anesthesia over the last two decades. Liposomal formulations of local anesthetics, once dubbed the holy grail of regional anesthesia, are one of the facets of such exploration. ${ }^{1}$ For decades, anesthesiologists have traditionally used continuous catheter techniques to prolong the duration of postoperative analgesia, ${ }^{2}$ but nerve block catheters are not without problems. Secondary failure is not uncommon for continuous catheters and, while such failures have decreased with the use of ultrasound guidance, they are still a pertinent problem. ${ }^{3}$ Additionally, continuous infusion of nerve blocks are relatively resource-intensive as they require added equipment, training, and monitoring. ${ }^{4}$ Hence, efforts to selectively prolong sensory blockade without the need for a continuous local anesthetic infusion have been gaining traction. Such attempts include the exploration of perineural adjuvants (dexmedetomidine, dexamethasone and clonidine), ${ }^{5}$ the development of novel drugs resulting in selective sodium channel blockade (saxitoxin and neosaxitoxin) ${ }^{6,7}$ and last, but not least, liposomal formulation of local anesthetics.

Liposomal formulations of medications are widely adopted in clinical medicine as they offer several advantages such as increased efficacy, decreased toxicity,

Y. S. Ranganath, MBBS MD

Department of Anesthesia, Roy J and Lucille A. Carver College of Medicine, University of Iowa, Iowa City, IA, USA

R. V. Sondekoppam, MBBS MD ( $₫)$

Department of Anesthesia, University of Iowa Hospitals and

Clinics, 200 Hawkins Dr, Iowa city, IA, USA

e-mail: rakesh-sondekoppam@uiowa.edu sustained therapeutic effects at the target site, and an increased therapeutic index. In theory, a liposomal formulation of a long-acting local anesthetic deposited close to the surgical field or its congruent terminal innervation should provide prolonged analgesic benefit with minimal motor or autonomic side effects. Liposomal bupivacaine (LB), first of the many candidate sustainedrelease local anesthetic formulations, has been popularized in the last decade ever since the U.S. Food and Drug Administration approved its use for postoperative pain in 2011. ${ }^{\mathrm{A}}$ Its use was subsequently expanded to include transversus abdominis plane (TAP) blocks in 2015 and interscalene blocks for shoulder surgery in 2018. ${ }^{\mathrm{B}, \mathrm{C}}$ While LB seemed an attractive option to regional anesthesiologists by offering the prospect of prolonged analgesia similar to continuous catheter techniques with the convenience of single injection, the clinical evidence has not shown unequivocally superior analgesic benefits over unencapsulated local anesthetic solutions.

In this issue of the Journal, Fidkowski et al. ${ }^{8}$ report on one such comparison of the analgesic properties of LB, unencapsulated bupivacaine hydrochloride $(\mathrm{BH})$, or their combination when administered via TAP blocks for postoperative pain control after open abdominal hysterectomies. This three-arm, single centre, randomized trial included two primary outcomes and multiple secondary outcomes; they did not find a statistically or clinically significant difference in any of the outcomes between the three study groups. The findings of the study are in contrast to two previous randomized-controlled trials reporting decreased pain scores and opioid requirements for up to $72 \mathrm{hr}$ after robotic-assisted hysterectomy and laparoscopic hand-assisted donor nephrectomy with the use of LB compared with BH., ${ }^{9,10}$ These two earlier studies have been critiqued for methodological and reporting 
TABLE Non-analgesic benefits to consider when analgesic modalities show non-inferiority

Impact on ambulation and quality of recovery

Impact on length of stay

Ease of administration

Impact on opioid dispensing at discharge

Resource utilization (human resources, drugs, equipment)

limitations (such as a lack of pre-enrollment study registration, lack of specification of primary outcome measures, and lack of correction for multiple comparisons); similarly, the present study by Fidkowski et al. ${ }^{8}$ reporting on the lack of benefit with LB also suffers from several methodological and reporting issues that may make the results less persuasive.

Studies supporting the benefit of $\mathrm{LB}^{11}{ }^{13}$ or refuting its utility in comparison with $\mathrm{BH}$ exist ${ }^{14}$ and the reasons for such discrepancy are multifaceted. Firstly, the severity of pain following different types of surgeries differs and may explain why LB has shown to have lower pain scores than $\mathrm{BH}$ following minor surgeries such as hemorrhoidectomies and bunion surgeries but may not be impactful in lessening the pain following major surgeries such as total knee arthroplasty. A similar trend may be present for pain following hysterectomies where LB has shown benefit following laparoscopic approaches but, as shown in the present study, may not be better than $\mathrm{BH}$ following open procedures. Secondly, liposomal formulations are known to have variable in vitro and in vivo stability, thereby affecting the pharmacokinetics of these formulations and subsequently their efficacy. ${ }^{15,16}$ But before making any such conclusions from the present study, it is worth noting that the investigators chose a subcostal TAP block approach (one often used for upper abdominal surgeries) for a lower abdominal surgical incision and, hence, the incongruency of the surgical incision with that of the block cover may in itself explain the lack of difference between the three groups. A classical or lateral TAP block may have been a better choice as the incisions were predominantly infra-umbilical.

To quote Cohen, "The primary product of a research inquiry is one or more measures of effect size, not $P$ values" 17 but the present study did not demonstrate either substantive clinical significance or statistical significance for any outcome measures. Like any study showing a lack of difference in outcome measures, the present study will also likely be scrutinized for the possibility of type II error. The authors had hypothesized a moderate effect size of $35 \%$ and $40 \%$ for their primary outcomes (total opioid consumption during the first 72 postoperative hours and the time to first opioid analgesic use, respectively) and, while the present study may be right that there is no such moderate effect size, this still does not rule out the possibility of a smaller effect size. The latter may only be apparent once more patients are studied.

Issues in the present study with regards to the choice of block and a reasonable probability of type II error may make the study findings less reliable, but this study still raises an important question. If the current evidence on decreasing pain scores following infiltration or field analgesia with LB is, at best, equivocal, ${ }^{18}{ }^{20}$ one may wonder if there is any role for the use of LB in clinical practice. Continuous catheter techniques have shown superior analgesia than single-injection regional techniques or local infiltration using unencapsulated local anesthetics, ${ }^{21}{ }^{23}$ but such practices may not be universally adaptable, nor are they without caveats. While the analgesic benefits of LB in a variety of surgeries still needs to be explored, it is equally important to know whether significant non-analgesic benefits exist. One such example is a recent meta-analysis of LB vs femoral nerve block (FNB), which showed no significant advantages of LB over FNB in terms of postoperative pain scores but showed a favourable impact of LB in reducing the length of stay and an opioid-sparing effect following total knee arthroplasty. ${ }^{24}$ If LB shows a non-inferior analgesic profile compared with other established modalities such as continuous nerve or interfascial blocks, there may still a potential case to be made for its use if it exhibits significant non-analgesic benefits (Table). ${ }^{25}{ }^{27}$

Are liposomal formulations of local anesthetics the holy grail of regional anesthesia to prolong the duration of analgesia we have been looking for? Sadly, the current evidence tells us this will not be the case as well-designed and well-conducted studies point towards equivalency of LB to single injection regional anesthetic blocks. The story of LB seems to emphasize once again just how vital it is to accumulate evidence on novel techniques from welldesigned pragmatic studies before their widespread clinical adoption. As rightly concluded in the most recent Cochrane review on the use of LB, while there is a lack of data to support or refute its use, further research is very likely to have an important impact. ${ }^{17}$

\section{La bupivacaïne liposomale pour prolonger l'analgésie des blocs TAP - deux pas en avant, un pas en arrière}

Depuis plus de deux décennies, les stratégies visant à prolonger de manière sélective la durée d'un bloc sensitif font l'objet à la fois de controverses et d'un intérêt soutenu 
TABLEAU Avantages non analgésiques à considérer lorsque les modalités analgésiques montrent une non-infériorité

Impact sur la déambulation et la qualité de la récupération Impact sur la durée d'hospitalisation

Facilité d'administration

Impact sur la prescription d'opioïdes au congé

Utilisation des ressources (ressources humaines, médicaments, équipement)

en anesthésie régionale. Les formulations liposomales d'anesthésiques locaux, autrefois considérées comme le Saint Graal de l'anesthésie régionale, sont l'une des facettes de cette exploration. ${ }^{1}$ Depuis des décennies, les anesthésiologistes ont traditionnellement utilisé des techniques avec perfusion continue pour prolonger la durée de l'analgésie postopératoire, ${ }^{2}$ mais les cathéters pour les blocs nerveux ne sont pas sans problème. Les défaillances secondaires ne sont pas rares en cas de perfusion continue et, tandis que de telles défaillances ont diminué avec l'utilisation de l'échoguidage, elles demeurent un problème d'actualité. ${ }^{3}$ En outre, la perfusion continue lors d'un bloc nerveux peut être très exigeante en matière de ressources car elle nécessite du matériel, de la formation, et du monitorage supplémentaires. ${ }^{4}$ Par conséquent, les efforts visant à prolonger de manière sélective le bloc sensitif sans avoir besoin d'une perfusion continue d'anesthésique local se sont imposés. Ces tentatives comprennent l'exploration d'adjuvants périneuraux (dexmédétomidine, dexaméthasone et clonidine) ${ }^{5}$ le développement de nouveaux médicaments entraînant un blocage sélectif des canaux sodiques (saxitoxine et néo-saxitoxine) ${ }^{6,7}$ et enfin, last but not least, les anesthésiques locaux en formulation liposomale.

Les formulations liposomales de médicaments sont largement adoptées en médecine clinique car elles offrent plusieurs avantages tels qu'une efficacité accrue, une toxicité réduite, des effets thérapeutiques soutenus au site cible et un indice thérapeutique augmenté. En théorie, une formulation liposomale d'un anesthésique local à action prolongée déposé près du champ chirurgical ou de son innervation terminale congruente devrait fournir des bienfaits analgésiques prolongés avec des effets secondaires moteurs ou autonomes minimaux. La bupivacaïne liposomale, la première des nombreuses formulations candidates d'anesthésiques locaux à libération soutenue, a été popularisée au cours de la dernière décennie après l'approbation de son utilisation pour le traitement de la douleur postopératoire par la Food and Drug Administration des États-Unis en 2011. ${ }^{\text {A }}$ Son utilisation a ensuite été élargie pour inclure les blocs des muscles du plan transverse de l'abdomen (blocs TAP) en 2015 et les blocs interscaléniques pour la chirurgie de l'épaule en 2018. ${ }^{\mathrm{B}, \mathrm{C}}$ Tandis que la bupivacaïne liposomale semblait être une option séduisante pour les anesthésiologistes régionaux car elle offrait la perspective d'une analgésie prolongée semblable aux techniques de perfusion continue mais avec l'avantage d'une injection unique, les données probantes cliniques n'ont pas montré d'avantages analgésiques résolument supérieurs par rapport aux solutions anesthésiques locales non encapsulées.

Dans ce numéro du Journal, Fidkowski et coll. ${ }^{8}$ rapportent les conclusions de leur comparaison des propriétés analgésiques de la bupivacaïne liposomale, du chlorhydrate de bupivacaïne non encapsulé, et de la combinaison de ces deux agents lorsqu'ils étaient administrés par blocs TAP pour le contrôle de la douleur postopératoire après une hystérectomie abdominale par laparotomie. Cette étude randomisée monocentrique à trois bras comptait deux critères d'évaluation principaux et de multiples critères secondaires; les auteurs n'ont trouvé aucune différence statistiquement ou cliniquement significative pour aucun de leurs critères entre les trois groupes à l'étude. Les résultats de l'étude détonnent avec deux études randomisées contrôlées antérieures qui faisaient état d'une diminution des scores de douleur et des besoins en opioïdes jusqu'à 72 heures après une hystérectomie robotisée et une néphrectomie conventionnelle de donneur par laparoscopie lors de l'utilisation de bupivacaïne liposomale par rapport au chlorhydrate de bupivacaïne.,10 Ces deux études antérieures ont été critiquées pour leurs limitations en matière de méthodologie et de communication des résultats (comme le manque d'enregistrement préalable de l'étude avant le recrutement, l'absence de spécification des critères d'évaluation principaux et l'absence de correction pour tenir compte des comparaisons multiples); de la même manière, l'étude de Fidkowski et coll. ${ }^{8}$ qui rapporte l'absence d'avantages de la bupivacaïne liposomale souffre elle aussi de plusieurs problèmes méthodologiques et de communication, ce qui pourrait rendre leurs résultats moins convaincants.

Il existe des études appuyant les avantages de la bupivacaïne liposomale ${ }^{11}{ }^{13}$ et d'autres réfutant son utilité par rapport au chlorhydrate de bupivacaïne, ${ }^{14}$ et les raisons de cette discordance sont multiples. Tout d'abord, l'importance de la douleur à la suite de différents types de chirurgie diffère et pourrait expliquer pourquoi la bupivacaïne liposomale a entraîné des scores de douleur inférieurs au chlorhydrate de bupivacaïne à la suite d'interventions chirurgicales mineures telles que les hémorroïdectomies et les chirurgies de l'oignon plantaire (halgus valgus), mais pourrait ne pas être aussi efficace pour diminuer la douleur à la suite d'interventions 
chirurgicales majeures telles qu'une arthroplastie totale du genou. Une tendance similaire pourrait être observée en ce qui touche à la douleur subséquente aux hystérectomies : en effet, la bupivacaïne liposomale a démontré des bienfaits à la suite d'une approche par laparoscopie mais, comme dans la présente étude, elle semble ne pas être supérieure au chlorhydrate de bupivacaïne après une intervention par laparotomie. Deuxièmement, nous savons que les formulations liposomales ont une stabilité in vitro et in vivo variable, affectant ainsi la pharmacocinétique de ces formulations et, par conséquent, leur efficacité. ${ }^{15,16}$ Mais avant de tirer de telles conclusions à partir de la présente étude, il convient de noter que les chercheurs ont choisi ici une technique de bloc TAP sous-costale (une approche souvent utilisée pour les chirurgies abdominales supérieures) pour une incision chirurgicale abdominale inférieure; par conséquent, l'absence d'adéquation entre l'incision chirurgicale et la couverture du bloc pourrait elle aussi expliquer l'absence de différence entre les trois groupes. Un bloc TAP classique ou latéral pourrait avoir été un meilleur choix étant donné que les incisions étaient principalement sous-ombilicales.

Pour citer Cohen, «La finalité primaire d'une question de recherche consiste en une ou plusieurs mesures de la taille d'effet, et non en valeurs $P{ }^{17}$; la présente étude n'a cependant démontré ni signification clinique ni signification statistique substantielle et ce, pour aucune mesure de ses résultats. Comme toute étude démontrant l'absence de différence dans les mesures des résultats, l'étude présentée ici sera également susceptible d'être évaluée à la recherche d'une possible erreur de type II. Les auteurs avaient émis l'hypothèse d'une taille d'effet modérée de $35 \%$ et $40 \%$ pour leurs critères d'évaluation principaux (consommation totale d'opioïdes au cours des 72 premières heures postopératoires et délai jusqu'à la première utilisation analgésique d'opioïdes, respectivement) et, bien que leur étude puisse avoir raison de conclure qu'il n'y a pas de taille d'effet modérée, cela n'exclut pas pour autant la possibilité d'une plus petite taille d'effet. Cette possibilité pourrait ne devenir apparente que lorsqu'un plus grand nombre de patients seront étudiés.

Les problèmes potentiels relevés dans la présente étude en ce qui concerne le choix du bloc et une probabilité raisonnable d'erreur de type II pourraient rendre ses conclusions moins fiables, mais cette étude soulève tout de même une question importante. Si les données actuelles sur la diminution des scores de douleur à la suite d'une infiltration ou d'une analgésie du champ avec de la bupivacaïne liposomale sont, au mieux, équivoques, ${ }^{18}{ }^{20}$ on peut se demander si la bupivacaïne liposomale devrait avoir un quelconque rôle dans la pratique clinique. Une analgésie supérieure a été démontrée lors de l'utilisation de techniques de perfusion continue par rapport aux techniques régionales à injection unique ou aux infiltrations locales d'anesthésiques locaux non encapsulés, ${ }^{21}{ }^{23}$ mais de telles pratiques pourraient ne pas être universellement adaptables et comportent leur propres risques. Alors que les avantages analgésiques de la bupivacaïne liposomale dans diverses interventions chirurgicales doivent encore être explorés, il est tout aussi important de savoir si des avantages non analgésiques existent. C'est le cas, par exemple, d'une méta-analyse récente comparant la bupivacaïne liposomale à un bloc du nerf fémoral (BNF), qui n'a montré aucun avantage significatif de la bupivacaïne sur le BNF en termes de scores postopératoires de douleur, mais a montré un impact favorable de la bupivacaïne liposomale pour réduire la durée de séjour et un effet d'épargne opioïde suivant une arthroplastie totale du genou. ${ }^{24} \mathrm{Si}$ la bupivacaïne démontre un profil analgésique non inférieur par rapport à d'autres modalités établies telles que les blocs nerveux continus ou les blocs interfasciaux, son utilité pourrait être confirmée si elle présentait d'importants avantages non analgésiques (tableau). ${ }^{25}{ }^{27}$

Les formulations liposomales d'anesthésiques locaux sont-elles alors le Saint Graal de l'anesthésie régionale pour prolonger la durée de l'analgésie que nous recherchions? Malheureusement, les données probantes actuelles nous indiquent que ce n'est pas le cas, car des études bien conçues et bien menées indiquent l'équivalence de la bupivacaïne liposomale à des blocs anesthésiques régionaux à injection unique. L'histoire de la bupivacaïne liposomale semble souligner une fois de plus à quel point il est essentiel d'accumuler des données probantes sur les techniques nouvelles à partir d'études pragmatiques bien conçues avant leur adoption clinique généralisée. Comme l'a conclu à juste titre la plus récente revue Cochrane sur l'utilisation de la bupivacaïne liposomale, bien qu'il manque de données pour appuyer ou rejeter son utilisation, d'autres recherches seront très susceptibles d'avoir un impact important. ${ }^{17}$

\section{Footnotes}

A. Pacira Pharmaceuticals, Inc. U.S. FDA approval of Exparel $^{\mathrm{TM}}$ for postsurgical pain management. Available from URL: https://investor.pacira.com/ news-releases/news-release-details/pacira-pharmaceuti cals-inc-announces-us-fda-approval-expareltm (accessed November 2020).

B. Janet Woodcock; Center for Drug Evaluation and Research Food and Drug Administration. Attn: David Stack. Chief Executive Officer \& Chairman. Letter to 
Pacira Pharmaceuticals, Inc. 2015. Available from URL: $\quad$ https://www.fda.gov/media/95042/download (accessed November 2020).

\section{U.S. Food and Drug Administration; T. Rabin. FDA In} Brief: FDA approves new use of exparel for nerve block pain relief following shoulder surgeries. April 6, 2018. Available from URL: https://www.fda.gov/ news-events/fda-brief/fda-brief-fda-approves-new-useexparel-nerve-block-pain-relief-following-shoulder-sur geries (accessed November 2020).

\section{Disclosures None.}

Funding statement None.

Editorial responsibility This submission was handled by Dr. Philip M. Jones, Associate Editor, Canadian Journal of Anesthesia.

Déclaration Aucune.

Déclaration de financement Aucune.

Editorial responsibility Cet article a été traité par Dr Philip M. Jones, rédacteur adjoint, Journal canadien d'anesthésie.

\section{References}

1. Grant SA. The Holy Grail: long-acting local anaesthetics and liposomes. Best Pract Res Clin Anaesthesiol 2002; 16: 345-52.

2. Ilfeld $B M$. Continuous peripheral nerve blocks: an update of the published evidence and comparison with novel, alternative analgesic modalities. Anesth Analg 2017; 124: 308-35.

3. Ahsan ZS, Carvalho B, Yao J. Incidence of failure of continuous peripheral nerve catheters for postoperative analgesia in upper extremity surgery. J Hand Surg Am 2014; 39: 324-9.

4. Jeng CL, Torrillo TM, Rosenblatt MA. Complications of peripheral nerve blocks. Br J Anaesth 2010; 105: i97-107.

5. Albrecht E, Kern C, Kirkham KR. A systematic review and metaanalysis of perineural dexamethasone for peripheral nerve blocks. Anaesthesia 2015; 70: 71-83.

6. Lobo K, Donado C, Cornelissen L, et al. A phase 1, doseescalation, double-blind, block-randomized, controlled trial of safety and efficacy of neosaxitoxin alone and in combination with $0.2 \%$ bupivacaine, with and without epinephrine, for cutaneous anesthesia. Anesthesiology 2015; 123: 873-85.

7. Rathmell JP, Strichartz G, Wanderer J. Neosaxitoxin versus traditional local anesthetics: mechanism of action and sites of notable effect. Anesthesiology 2015. https://doi.org/10.1097/01. anes.0000470956.02665.a6.

8. Fidkowski CW, Choksi N, Alsaden MR. A randomized-controlled trial comparing liposomal bupivacaine, plain bupivacaine, and the mixture of liposomal bupivacaine and plain bupivacaine in transversus abdominus plane block for postoperative analgesia for open abdominal hysterectomies. Can J Anesth 2021. https:// doi.org/10.1007/s12630-020-01911-1.

9. Hutchins J, Delaney D, Vogel RI, et al. Ultrasound guided subcostal transversus abdominis plane (TAP) infiltration with liposomal bupivacaine for patients undergoing robotic assisted hysterectomy: a prospective randomized controlled study. Gynecol Oncol 2015; 138: 609-13.

10. Hutchins JL, Kesha $R$, Blanco F, Dunn $T$, Hochhalter $R$. Ultrasound-guided subcostal transversus abdominis plane blocks with liposomal bupivacaine vs. non-liposomal bupivacaine for postoperative pain control after laparoscopic hand-assisted donor nephrectomy: a prospective randomised observer-blinded study. Anaesthesia 2016; 71: 930-7.

11. Onel E MH, Patou G, White PF. Exparel ${ }^{\mathrm{TM}}$, a liposomal bupivacaine local analgesic, extends pain relief and decreases opioid use. Anesthesiology 2010: A1158 (abstract).

12. Golf M, Daniels SE, Onel E. A phase 3, randomized, placebocontrolled trial of DepoFoam ${ }^{\circledR}$ bupivacaine (extended-release bupivacaine local analgesic) in bunionectomy. Adv Ther 2011; 28: 776-88.

13. Clinical trial no. SKY0402-C-210. A randomized, double-blind, active-control study to evaluate the safety and efficacy of a single local administration of SKY0402 for prolonged postoperative analgesia in subjects undergoing augmentation mammoplasty. Pacira Pharmaceuticals Inc.; Latest information update 2013.

14. Bagsby DT, Ireland PH, Meneghini RM. Liposomal bupivacaine versus traditional periarticular injection for pain control after total knee arthroplasty. J Arthoplast 2014; 29: 1687-90.

15. Narang AS, Chang RK, Hussain MA. Pharmaceutical development and regulatory considerations for nanoparticles and nanoparticulate drug delivery systems. J Pharm Sci 2013; 102: 3867-82.

16. Sercombe L, Veerati T, Moheimani F, Wu SY, Sood AK, Hua S. Advances and challenges of liposome assisted drug delivery. Front Pharmacol 2015. https://doi.org/10.3389/fphar.2015.00286.

17. Cohen J. Things I have learned (so far). American Psychologist 1990; 45: 1304-12.

18. Abildgaard JT, Chung AS, Tokish JM, Hattrup SJ. Clinical efficacy of liposomal bupivacaine: a systematic review of prospective, randomized controlled trials in orthopaedic surgery. JBJS Rev 2019. https://doi.org/10.2106/JBJS.RVW.18. 00192.

19. Hamilton TW, Athanassoglou V, Trivella M, Strickland LH, Mellon S, Murray D. Liposomal bupivacaine peripheral nerve block for the management of postoperative pain. Cochrane Database Syst Rev 2016. https://doi.org/10.1002/14651858. CD011419.pub2.

20. Raman S, Lin M, Krishnan $N$. Systematic review and metaanalysis of the efficacy of liposomal bupivacaine in colorectal resections. J Drug Assess 2018; 7: 43-50.

21. Bingham $A E, F u \quad R$, Horn JL, Abrahams MS. Continuous peripheral nerve block compared with single-injection peripheral nerve block: a systematic review and meta-analysis of randomized controlled trials. Reg Anesth Pain Med 2012; 37: 583-94.

22. Vorobeichik L, Brull R, Abdallah FW. Evidence basis for using perineural dexmedetomidine to enhance the quality of brachial plexus nerve blocks: a systematic review and meta-analysis of randomized controlled trials. Br J Anaesth 2017; 118: 167-81.

23. Malik T, Mass D, Cohn S. Postoperative analgesia in a prolonged continuous interscalene block versus single-shot block in outpatient arthroscopic rotator cuff repair: a prospective randomized study. Arthroscopy 2016; 32(1544-50): e1.

24. Liu Y, Zeng JF, Zeng $Y, W u Y G$, Bao XC, Shen B. Comprehensive comparison of liposomal bupivacaine with femoral nerve block for pain control following total knee arthroplasty: an updated systematic review and meta-analysis. Orthop Surg 2019; 11: 943-53.

25. Torgeson $M$, Kileny $J$, Pfeifer $C$, Narkiewicz L, Obi S. Conventional epidural vs transversus abdominis plane block 
with liposomal bupivacaine: a randomized trial in colorectal surgery. J Am Coll Surg 2018; 227: 78-83.

26. Butz DR, Shenaq DS, Rundell VL, et al. Postoperative pain and length of stay lowered by use of exparel in immediate, implantbased breast reconstruction. Plast Reconstr Surg Glob Open 2015. https://doi.org/10.1097/GOX.0000000000000355.

27. Dominguez DA, Ely $S$, Bach C, Lee T, Velotta JB. Impact of intercostal nerve blocks using liposomal versus standard bupivacaine on length of stay in minimally invasive thoracic surgery patients. J Thorac Dis 2018; 10: 6873-9.

Publisher's Note Springer Nature remains neutral with regard to jurisdictional claims in published maps and institutional affiliations. 